\title{
Evaluation of the male with chronic prostatitis/chronic pelvic pain syndrome
}

\section{R. Christopher Doiron, MD; J. Curtis Nickel, MD, FRCSC}

Department of Urology, Queen's University, Kingston, ON, Canada

Cite as: Can Urol Assoc J 2018;12(6Supp|3):S152-4. http://dx.doi.org/10.5489/cuaj.5322

\section{Introduction}

Chronic prostatitis/chronic pelvic pain syndrome (CP/CPPS) is a symptom complex associated with chronic pelvic pain in men after the exclusion of other confusable diseases, such as infection, malignancy, and benign prostatic hyperplasia $(\mathrm{BPH})$. Prostatitis symptoms is a common reason for outpatient referral to the urologist, as up to $9 \%$ of Canadian men will experience some type of prostatitis symptoms (1/3 of these will report very bothersome symptoms to their physician) over the course of a year. These cases can constitute up to $2 \%$ of a urologist's outpatient practice.

A proper diagnosis and evaluation in these men uses our general urologic skills and tools available to us in the outpatient clinic. The evaluation and workup need not be overly complex, but rather follow an organized approach while keeping in mind a few general principles that will aid in organizing an efficient and potentially successful management strategy.

\section{Practice tips}

\section{Tip 1: Keep evaluation simple}

Use the basic urologic principles of history (talk to the patient), physical exam (examine the patient, including the prostate and pelvic floor), and laboratory evaluation (urinalysis and urine culture) available in outpatient clinic — don't be afraid to be a clinician! Managing these patients in a busy outpatient urologist's office is doable and should not be more onerous than management of a $\mathrm{BPH}$ or overactive bladder $(\mathrm{OAB})$ patient.

\section{Tip 2: Does the patient have symptoms of CP/CPPS?}

Symptoms include:

- Chronic pelvic pain in: perineum, testicles, tip of penis or below waist in pubic or bladder area
- Has it been present for at least three months?

Note: Unilateral orchalgia is not CP/CPPS; please see supplement publication on "Chronic scrotal contents pain" for further discussion of this condition.

Tip 3: Have you ruled out confusable and/or associated diseases?

These may include:

- Urinary tract infection (UTI) and bacterial prostatitis: History, urine culture

- BPH: Symptoms and size of prostate

- OAB: Storage symptoms with no pain

- Pelvic floor dysfunction: Trigger point or myofascial pain/spasm

- Malignancy: Urinalysis, cytology, +/- cystoscopy

- Determine contribution of pelvic floor dysfunctional pain: Physical exam

- Calculi: Nature of pain, urinalysis

- Interstitial cystitis/bladder pain syndrome (IC/BPS): Pain with bladder filling, relief with emptying

- Irritable bowel syndrome (IBS): Absominal/pelvic pain associated with diarrhea $+/$ - constipation

Tip 4: Determine the clinical picture using the UPOINT phenotype tool

CP/CPPS is a heterogeneous patient population; identifying the patient phenotype is of utmost importance and will greatly impact management plan and outcomes. The UPOINT tool allows identification of up to six clinically defined domains and will help simplify the approach for the practitioner.

Tip 5: Quantitate symptoms using the National Institutes of Health Chronic Prostatitis Symptom Index (NIH-CPSI) tool (Fig. 1)

This simple, one-page index can be completed by patients in minutes during their outpatient visits and includes everything you need to know about their disease: location of pain, frequency, pain severity, urinary symptoms, and impact of symptoms on quality of life. This can be a useful tool to monitor progress and assess objective response to management plans. 


\section{NIH-Chronic Prostatitis Symptom Index}

\section{Pain or discomfort}

1. In the last week, have you experienced any pain

or discomfort in the following areas?

$\begin{array}{lcc} & \text { Yes } & \text { No } \\ \text { a. Area between rectum and testicles (perineum) } & \square 1 & \square 2 \\ \text { b. Testicles } & \square 1 & \square 2 \\ \text { c. Tip of the penis (not related to urination) } & \square 1 & \square 2 \\ \text { d. Below your waist, in your pubic or bladder area } & \square 1 & \square 2\end{array}$

2. In the last week, have you experienced:

a. Pain or burning during urination?

b. Pain or discomfort during or after sexual climax $\quad \square 1 \quad \square 2$ (ejaculation)?

3. How often have you had pain or discomfort in any of these areas over the last week?

\section{$\square$ Never \\ $\square$ Rarely \\ ○ 2 Sometimes \\ ๑ 3 Often \\ $\square 4$ Usually \\ a Always}

4. Which number best describes your AVERAGE pain or discomfort on the days that you had it, over the last week?

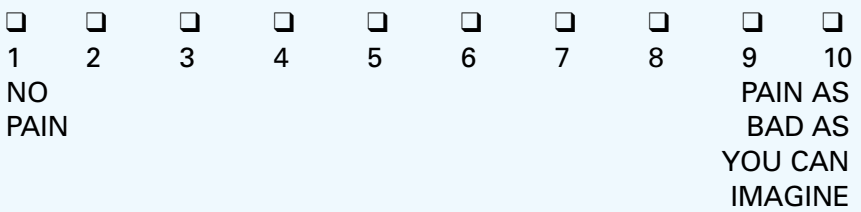

\section{Urination}

5. How often have you had a sensation of not emptying your bladder completely after you finished urinating, over the last week?

0 Not at all

1 Less than 1 time in 5

$\square 2$ Less than half the time

3 About half the time

$\checkmark 4$ More than half the time

$\square$ Almost always

Tip 6: Examine the patient

It is important to determine specific pain locations for your CP/CPPS patients, and also to rule out other abdominal or pelvic pathology. A careful pelvic floor examination performed prior to actual prostate palpation allows one to examine trigger points and pelvic floor spasm and tenderness more accurately.
6. How often have you had to urinate again less than two hours after you finished urinating, over the last week?

\section{$\square 0$ Not at all}

a 1 Less than 1 time in 5

$\square 2$ Less than half the time

3 About half the time

4 More than half the time

$\square$ Almost always

\section{Impact of Symptoms}

7. How much have your symptoms kept you from doing the kinds of things you would usually do, over the last week?
$\square 0$ None
1 Only a little
$\square 2$ Some
$\square 3$ A lot

8. How much did you think about your symptoms, over the last week?
$\square$ None
$\square 1$ Only a little
2 Some
○ 3 A lot

\section{Quality of Life}

9. If you were to spend the rest of your life with your symptoms just the way they have been during the last week, how would you feel about that?
$\square$ Delighted
$\square 1$ Pleased
2 Mostly satisfied
\ 3 Mixed (about equally satisfied and dissatisfied)
$\checkmark$ Mostly dissatisfied
$\square$ Unhappy
a 6 Terrible

\section{Scoring the NIH-Chronic Prostatitis Symptom Index Domains}

Pain: Total of items 1a, 1b, 1c, 1d, 2a, 2b, 3, and $4=$

Urinary Symptoms: Total of items 5 and $6=$

Quality of Life Impact: Total of items 7, 8, and $9=$

Fig. 1. The NIH Chronic Prostatitis Symptom Index (CPSI) tool can be usefu to first evaluate and then secondarily monitor patients' progress and assess response to treatment (see "Recommended reading" for reference).

\section{Tip 7: Perform prostate massage}

Chronic bacterial infection of the prostate (category II) must be ruled out. This is best achieved with a pre- and postprostate massage urine test. Uropathogenic bacteria cultured in the post-prostate massage urine specimen or more colony counts in the post-prostatic massage specimen compared to the pre-prostate massage midstream urine specimen indi- 


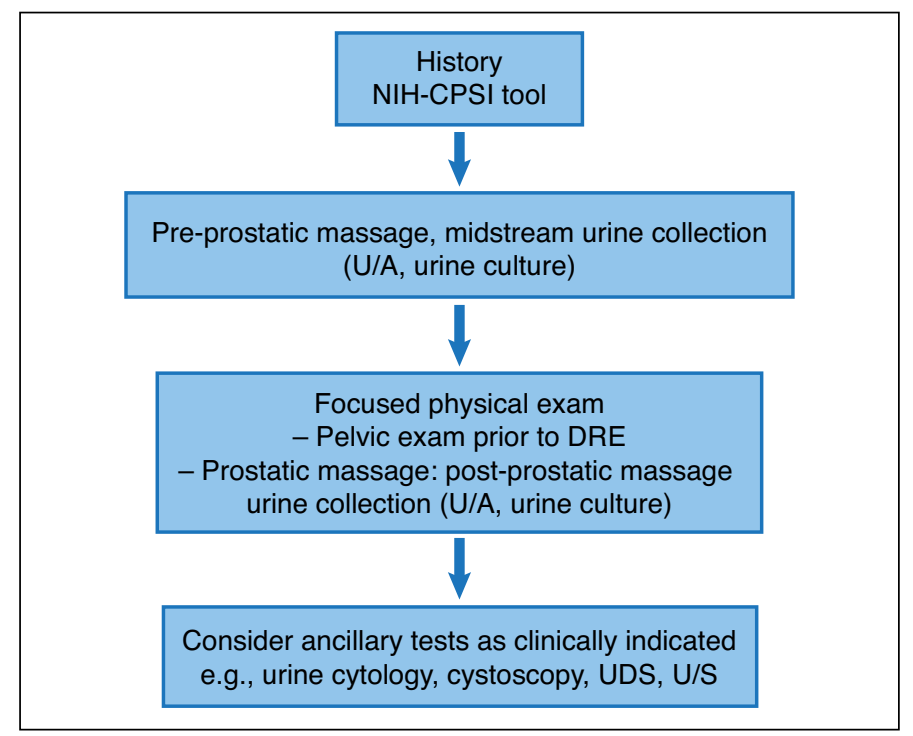

Fig. 2. A suggested evaluation algorithm for a patient presenting with chronic prostatitis/chronic pelvic pain syndrome. DRE: digital rectal exam; NIH-CPSI: National Institutes of Health Chronic Prostatitis Symptom Index; U/A: urinalysis; UDS: urodynamic studies; U/S: ultrasound.

cates that the patient may have a bacterial infection that could be managed with antimicrobial therapy.

\section{Tip 8: Ancillary tests}

Further ancillary tests, such as cystoscopy, urodynamics, and/or ultrasound (pelvic or transrectal) are indicated in selected patients:

- Failure to respond to treatment

- Complex cases

- Significant obstructive voiding symptoms

- Hematuria and/or suspicious urine cytology

- Pain referable to bladder (rule out IC/BPS, Hunner's lesion)

- Severe pain on ejaculation (ejaculatory or prostate cysts, seminal vesicle pathology can sometimes only be identified by ultrasound)
- Urodynamics can be useful primarily to evaluate possibility of obstruction, e.g., bladder neck or detrusor sphincter dyssynergia

\section{Summary}

An accurate diagnosis and assessment (Fig. 2) of the clinical picture of each patient will allow successful, individualized phenotype-directed therapy.

Competing interests: Dr. Nickel has been a consultant for Astellas, Auxillium, Eli Lilly, Farr Labs, Ferring, GSK, Pfizer, Redleaf Pharma, Taris Biomedical, Tribute, and Trillium Therapeutics; a lecturer for Astellas and Eli Lilly; and has participated in clinical trials supported by Eli Lilly, GSK, J\&J, Pfizer, and Taris Biomedical. Dr. Doiron reports no competing personal or financial conflicts related to this work.

This paper has been peer reviewed.

\section{Recommended reading}

1. Shoskes DA, Nickel JC, Dolinga R, et al. Clinical phenotyping of patients with chronic prostatitis/ chronic pelvic pain syndrome and correlation with symptom severity. Urology 2009;73:538-42. https://doi.org/10.1016/i.urology.2008.09.074

2. Litwin MS, McNaughton Collins M, Fowler MJ, et al. The NIH Chronic Prostatitis Symptom Index (NIH-CPSI): Development and validation of a new outcome measure. J Urol 1999;162:369-75. https://doi.org/10.1016/S0022-5347(05)68562-X

3. Nickel IC, Shoskes D, Wang Y, et al. How does the pre-massage and post-massage 2-glass test compare to the Meares-Stamey 4-glass test in men with chronic prostatitis/chronic pelvic pain syndrome? I Urol 2006;176:119-24. https://doi.org/10.1016/S0022-5347(06)00498-8

4. Nickel JC. Prostatitis. Can Urol Assoc J 2011;5:306-15. https://doi.org/10.5489/cuaj.686

Correspondence: Dr. R. Christopher Doiron, Department of Urology, Queen's University, Kingston, ON, Canada; chris.doiron@queensu.ca 